\title{
A Description-Processing System for Soccer Agents and NIT Stones 98
}

\author{
Nobuhiro Ito, Kouichi Nakagawa, Xiaoyong Du, and Naohiro Ishii \\ Department of Intelligence and Computer Science, Nagoya Institute of Technology, \\ Gokiso-cho, Showa-ku, Nagoya 466, JAPAN
}

\begin{abstract}
Many conventional object-oriented models suffer some problems in the representation of the multiple objects. A multiple object is an object with multiple aspects, autonomy, and pro-activeness. This paper proposes a new agent model called EAMMO, which applies the agent-oriented paradigm to represent multiple objects. EAMMO consists of three types of agents as follows: (1) an upper-agent describes autonomous objects, (2) a lower-agent describes reflective objects, and (3) an environmental-agent describes the environment including agents. We design a description-processing system for EAMMO to confirm the efficiency of our model. We describe soccer games in EAMMO. A soccer player is a good example for EAMMO, because a soccer player is a kind of multiple objects. We define only basic skills and strategies in the soccer agent. However, we found that soccer agents can corporate in a more complex strategy generated by the system. As the result, we confirm that EAMMO is an efficient model for describing multiple objects.
\end{abstract}

\section{Introduction}

It is an important problem in AI how an object collaborates with others to accomplish a goal in a dynamic environment. A good example is soccer games, where a soccer player need to collaborates with other teammates to win a game. Recently, much attention is paid to simulate soccer games on computer. A contest called the Robot Soccer World Cup (RoboCup) has been held two times. It has seen as a new landmark of AI research instead of chess games. In this paper, we pay special attention to such kinds of objects like soccer players. They have the following features:

1. multiple aspects: The object has different aspects (roles) in different time, places, and cases. For example, a soccer player has several possible roles like passer, shooter, or dribbler.

2. autonomy: The object can decide one of its multiple aspects by itself according to time, places, and cases. For example, a soccer player passes the ball if he keeps the ball and is near to a teammate. However, he shoots the ball if he is near enough to the opponent goal.

3. pro-activeness: The object can initial some behaviors by itself in a dynamic environment. For example, a soccer player can change his strategies according to time left. 
We call the objects with the above three features "multiple objects" in this paper. Informally,

Multiple object $=$ multiple aspects + autonomy + pro-activeness

To represent multiple aspects of objects, many concepts like views[2], aspects[3], roles[4], deputy[5], are proposed in existing object-oriented models. However, these models can not represent the autonomous and pro-active features of multiple objects. On the other hand, the existing agent models[6, 7, 8, 9] can represent autonomy and pro-activeness, appropriately. However, they omit the needs of multiple aspects of multiple objects.

In this paper, we propose a new agent model, called EAMMO(an Environmental Agent Model for Multiple Objects), to represent multiple objects. Our model consists of three type agents.

1. An upper-agent describes an autonomous object with multiple aspects. It has a status processor to decide its next state, and an action processor to decide actually its operation.

2. A lower-agent describes a reactive object, and has only one action processor to realize reactive behaviors.

3. An environmental-agent describes an autonomous and pro-active object. It manages the environment information which affects all agents in the environment.

We also implement an interpreter for the description language of EAMMO to confirm the effectiveness of our model. Furthermore, we describe soccer players in the language and simulate soccer games on the computer. From experiments, we found that the soccer players can corporate in a more complex strategy generated by the system itself than those strategies we defined initially for the soccer players. Thus, we confirm that EAMMO is an effective model for describing multiple objects (soccer players).

The paper is organized as follows: In Section 2, and Section 3, we introduce some basic concepts and describe the EAMMO and its description language. In Section 4, we explain an implementation of an interpreter for EAMMO. For evaluating the effectiveness of our model, we define three types of soccer players (multiple objects) in the language, and simulate the soccer games in Section 5. We also introduce our team(NIT Stones) of RoboCup-98 in the section. Finally, we conclude the paper in Section 6 .

\section{Basic Concepts}

We discuss basic concepts of our model in this section. The definition of EAMMO is given in the next section.

As we know, an object in conventional object-oriented models (we call it a regular object in this paper) consists of an identifier, a list of attributes, and a set of methods. The attributes show the internal status of a regular object. They are defined as follows. 
Definition 1. (Attribute) An attribute is a pair of attribute_name and attribute_value. That is.

$$
\langle\text { attribute }\rangle::=\langle\text { attribute_name }\rangle(\langle\text { attribute_value }\rangle)
$$

The list of attributes of an object can be specified as

$$
\text { Attributes }\{\langle\text { attribute }\rangle, \ldots,\langle\text { attribute }\rangle\}
$$

In the definitions, the words being from an uppercase are reserved words.

Definition 2 . (Identifier) An object always has a unique identifier.

$$
\langle\text { identifier }\rangle:=\text { Object }(\langle\text { object_name }\rangle)
$$

Definition 3 . (Method) A method is a pair of method_name and method_body.

$$
\langle\operatorname{method}\rangle::=\langle\text { method_name }\rangle\left(\left\langle\operatorname{method} \_ \text {body }\right\rangle\right)
$$

where 〈method_body is a procedure/function written in some languages. The set of methods of an object can be specified as

$$
\text { Methods }\{\langle\text { method }\rangle, \ldots,\langle\text { method }\rangle\}
$$

By using these definitions, a regular object can be defined as follows.

Definition 4 (Regular Object) A regular object consists of an identifier, a list of attributes, and a set of methods. That is,

$$
\begin{aligned}
\langle\text { regular_object }\rangle::= & 0 \text { bject }(\langle\text { object_name }\rangle), \\
& {[\text { Attributes }\{\langle\text { attribute }\rangle, \ldots,\langle\text { attributes }\rangle\},} \\
& {[\text { Methods }\{\langle\operatorname{method}\rangle, \ldots,\langle\operatorname{method}\rangle\}]] }
\end{aligned}
$$

A message is an import concept in communication among regular objects in the object-oriented paradigm. Our agents also communicate each other through messages.

Definition 5 . (Message) A message consists of a content, sender, and receiver of the message.

$$
\langle\text { message }\rangle::=\text { Message }(\langle\text { a_content_of_the_message }\rangle,\langle\text { sender }\rangle,\langle\text { receiver }\rangle)
$$

where 〈a_content_of_message is a method name or a keyword for invoking a method.

The multiple objects are defined by adding autonomy and pro-activeness to regular objects.

Our way is to define a status processor and an action processor, for a multiple object. By using these processors, a multiple object is able to decide its behavior autonomously through following two steps. 
1. By using the status processor, the object decides one of its multiple aspects by itself.

2. It then decides a method by the action processor.

Definition 6. (Status Processors) The status processor of an object is a process that modifies the attributes and methods of the object according to the relation with others, the environment, and its current status.

$$
\begin{gathered}
\langle\text { status_processor }\rangle:=\operatorname{Status}\{\langle\text { relations }\rangle,\langle\text { roles }\rangle\} \\
\langle\text { relations }\rangle:=(\langle\text { relation }\rangle, \ldots,\langle\text { relation }\rangle) \\
\langle\text { relation }\rangle:=\operatorname{Relation}(\langle\text { relation_name }\rangle) \leftarrow \text { object }(\langle\text { object_name }\rangle) \\
\langle\text { roles }\rangle::=(\langle\text { role }\rangle, \ldots,\langle\text { role }\rangle) \\
\langle\text { role }\rangle::=\langle\text { attribute_name }\rangle(\langle\text { attribute_value }\rangle) \leftarrow \operatorname{Relation}(\langle\text { relation_name }\rangle), \\
\quad \text { Own }(\langle\text { attributes }\rangle), \\
\operatorname{Env}(\langle\text { attributes }\rangle) \\
\operatorname{Own}(\langle\text { attributes }\rangle)::=\operatorname{Own}\{\langle\text { attribute }\rangle, \ldots,\langle\text { attribute }\rangle\} \\
\operatorname{Env}(\langle\text { attributes }\rangle)::=\operatorname{Env}\{\langle\text { attribute }\rangle, \ldots,\langle\text { attribute }\rangle\}
\end{gathered}
$$

where Status shows that the followings are rules to decide its own status. Relation shows that the followings are rules to restrict its relations between the object and others. Own shows that the attributes are the current own status(attributes). Env shows that the attributes are the current status of the environment. Own and Env are represented by a list of attributes.

The arrow in 〈relation〉 means that if the object in the right-hand is appeared, the relation denoted by 〈relation_name $\rangle$ occurs. Similarly, the arrow in $\langle$ role $\rangle$ means that if the conditions in the right-hand are satisfied the attribute

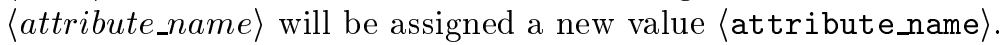

Example 1. We define a status process for the object "Ito".

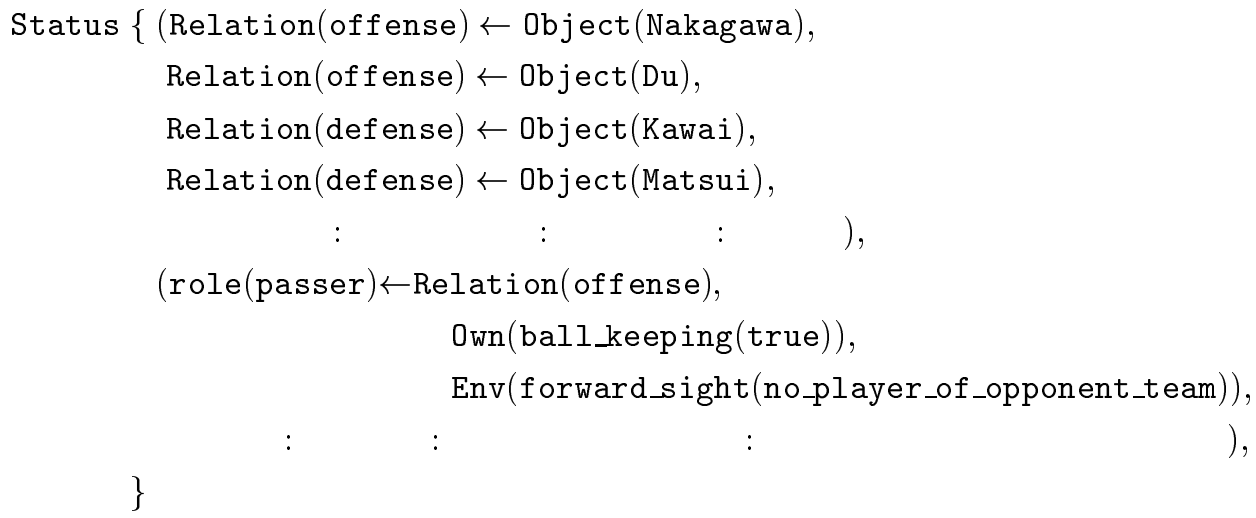

It means that "Ito" is in the relation "offense" if the object "Nakagawa" or "Du" (who is the member of own team) is detected by himself. He is in relation 
"defense" if the object "Kawai" or "Matsui" (who is the member of opponent team)is detected. If "Ito" is in the relation "offense", and he is keeping the ball and no player of the opponent team is in his forward sight, then he will change his attribute "role" into "passer".

Definition 7. (Action Processor) The action processor of an object is a process that select a proper method for the object according to decided status by the status processor, the environment, and the message that the object received.

$$
\begin{aligned}
\langle\text { action_processor }\rangle: & :=\operatorname{Actions}\{\langle\text { action }\rangle, \ldots,\langle\text { action }\rangle\} \\
\langle\text { action }\rangle:=(\langle\text { method_name }\rangle \leftarrow \operatorname{Own}(\langle\operatorname{attributes}\rangle) & \\
& \operatorname{Env}(\langle\text { attributes }\rangle), \\
& \operatorname{Msg}(\langle\text { a_content_of_the_message }\rangle))
\end{aligned}
$$

where Msg shows that the following 〈a_content_of_the_message is a message defined in Definition 5 . When the conditions in the right-hand of the arrow are satisfied, the method $\langle$ method_name $\rangle$ is invoked.

Example 2. One possible action processor for the object "Ito" is

$$
\begin{aligned}
\text { Actions }\{\text { pass } \leftarrow & 0 w n(\text { role }(\text { passer })) \\
& \text { Env(distance_to_the_goal }(50)) \\
& \text { Msg(pass }), \\
: \quad: & : \\
\} &
\end{aligned}
$$

It means that "Ito" will kick the ball to the goal if he has a role of passer and he is near to the goal, and he gets a message including pass in the content of the message.

\section{EAMMO}

EAMMO(an Environmental Agent Model for Multiple Objects) is a multi-agent model that we proposed for representing multiple objects.

By using a status processor and an action processor, we extend a regular object with autonomy and pro-activeness. These processors require "the environment" in their descriptions. In other words, representing a multiple object requires an environment. The environment has pro-activeness (like time) and autonomy (like up/down temperature). So, we can regard the environment as one kind of agents. In real-world, entities have different powers. Some behave complicatedly such as human beings, and others behave simply such as tools. Hence, when we use agents to model these entities, different types of agents with different powers are necessary. For example, the agents for tools do not always have autonomous and pro-activeness features, and the agents for men, to be opposite, need more power. Therefore, we classify agents in our model 


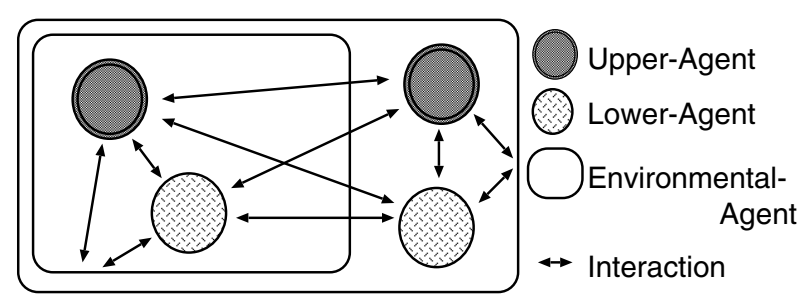

Fig. 1. Relationship among Three Types of Agents

to upper-agents who behave autonomously and pro-actively, and lower-agents who behave reactively, as well as environmental-agents who send messages to the other agents pro-actively. Figure.1 shows the relationship among these three types of agents. In Fig. 1, the environmental-agents include upper-agents and lower-agents. These three types of agents interact with each other and all of them are used to represent multiple objects.

\subsection{Lower-Agents}

\section{Definition 8 Lower-Agent.}

A lower-agent consists of an identifier, a list of attributes, a set of methods, and an action processor.

$$
\begin{aligned}
\langle\text { lower-agent }\rangle::= & \text { Agent }(\langle\text { agent_name }\rangle), \\
& {[\text { Attributes }\{\langle\text { attribute }\rangle, \ldots,\langle\text { attributes }\rangle\},} \\
& {[\text { Methods }\{\langle\text { method }\rangle, \ldots,\langle\text { method }\rangle\},} \\
& {[\text { Actions }\{\langle\text { action }\rangle, \ldots,\langle\text { action }\rangle\}]]] }
\end{aligned}
$$

This definition shows that a lower-agent consists of a regular object and an action processor. The concept of the lower-agent is shown in Fig. 2. The loweragent only behaves with messages from other agents, reactively. So, a single action processor deals with the attributes and the methods in Fig. 2.

\subsection{Upper-Agents}

Definition 9 . (Upper-Agent) An upper-agent is defined as an object that has multiple aspects, and behaves autonomously and pro-actively. An upperagent consists of an identifier, a list of attributes, a set of methods, a status processor, and an action processor.

$$
\begin{aligned}
\langle\text { upper-agent }\rangle:: & \operatorname{Agent}(\langle\text { agent_name }\rangle), \\
& {[\text { Attributes }\{\langle\text { attribute }\rangle, \ldots,\langle\text { attribute }\rangle\},} \\
& {[\text { Methods }\{\langle\text { method }\rangle, \ldots,\langle\text { method }\rangle\}}
\end{aligned}
$$




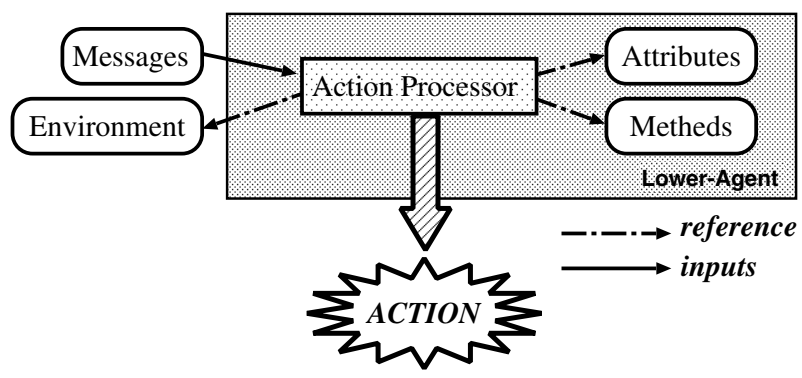

Fig. 2. A Lower-Agent

[Sequential_methods $\{\langle$ sequential_method $\rangle$, $\ldots,\langle$ sequential_method $\rangle\}]]]$,

[Status $\{\langle$ relatiosn $\rangle,\langle$ roles $\rangle\}]$,

[Actions $\{\langle$ aciton $\rangle, \ldots,\langle$ aciton $\rangle\}]$

where 〈sequential_method is a sequence of methods. It is defined as

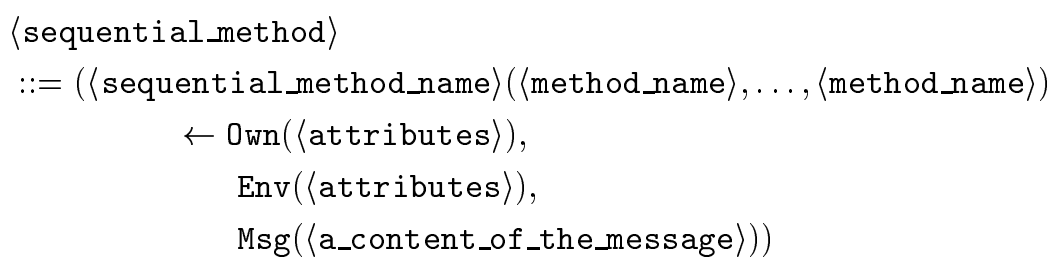

When a sequential method (sequential_method_name) is detected, the following $\langle$ method_name)s are invoked orderly.

A lower-agent plus a status processor constructs an upper-agent. The concept of the upper-agent is shown in Fig. 3. The status and action processors modify attributes and sequential methods, when the upper-agent receives messages. Since upper-agents have autonomous and pro-active features, these agents must modify attributes and sequential methods at runtime. "Messages" in Fig. 3 are the messages from other agents, including the environmental-agents. The status processor modifies the attributes and the sequential methods according to the status of the environment, the messages, and the attributes before modification. By modifying the attributes and the sequential methods with the status processor, the upper-agent decides next own status. Then, the action processor actually decides its behavior(a sequential method), using an output of the status processor.

\subsection{Environmental-Agents}

Definition 10 . (Environmental-Agent) An environmental-agent consists of an identifier, a list of attributes, a set of methods, a status processor, an action 




Fig. 3. An Upper-Agent

processor and an environmental database.

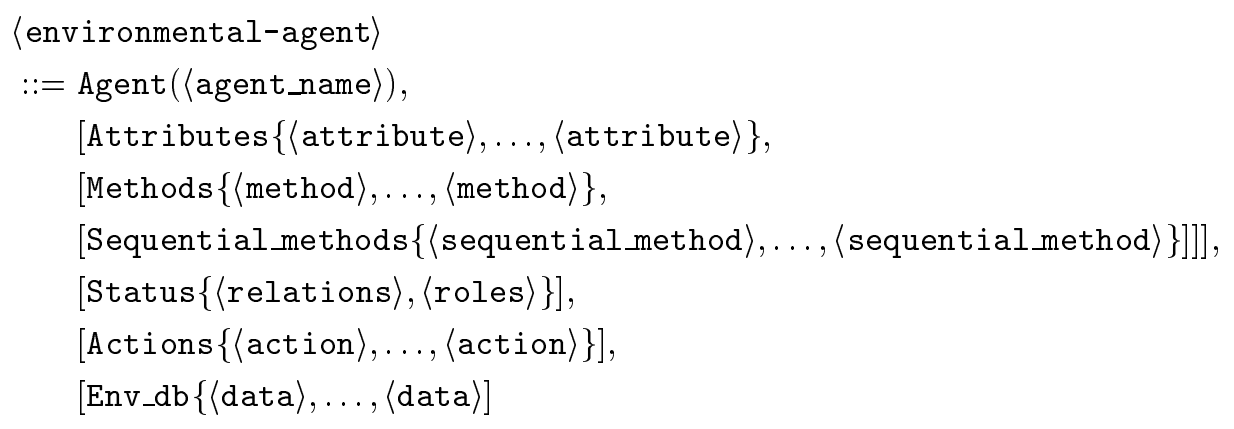

where Env_db is a reserved words, and means an environmental database. It is defined as

$$
\langle\text { environmental_database }\rangle::=\operatorname{Env} \_d b\{\langle\text { data }\rangle, \ldots,\langle\text { data }\rangle\}
$$

$\langle$ data $\rangle::=($ Agent $(\langle$ agent_name $\rangle), \operatorname{Attributes}\{\langle$ attribute $\rangle, \ldots,\langle$ attribute $\rangle\})$

An environmental-agent consists of an upper-agent and an environmental database. The concept of an environmental-agent is shown in Fig. 4. Upperagents and lower-agents do not have visible sensors. The environmental-agent plays a visual role for the other agents, and it provides other agents the visual data. The visual data are stored in the environmental database. The environmentalagent can multi-cast and broadcast to the agents with the same attribute values, by searching the environmental database for these attributes. When agents move from an environmental-agent to another environmental-agent, the result of the movement is registered in and deleted from the two environmental-databases respectively. 




Fig. 4. An Environmental-Agent

Table 1. Strategies for A Soccer Player

\begin{tabular}{|c|c|c|c|c|c|}
\hline Relation & \multicolumn{4}{|c|}{ offense } & defense \\
\hline Location & \multicolumn{2}{|c|}{ in goal area } & \multicolumn{2}{|c|}{ not in goal area } & - \\
\hline Ball & 0 & $x$ & 0 & $x$ & - \\
\hline Strategy & shoot & $\begin{array}{c}\text { go to } \\
\text { goal }\end{array}$ & pass & $\begin{array}{l}\text { go to } \\
\text { goal }\end{array}$ & $\begin{array}{c}\text { track } \\
\text { ball }\end{array}$ \\
\hline $\begin{array}{l}\text { exceptional } \\
\text { strategy }\end{array}$ & - & — & $\begin{array}{l}\text { Short distance to } \\
\text { the goal } \rightarrow \text { shoot }\end{array}$ & 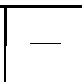 & - \\
\hline
\end{tabular}

\subsection{An Example}

Here, we consider a soccer player "Ito" who behaves under the strategies in Table 1. This is a good example to represent multiple objects, because a soccer player require some aspects with surroundings in the game. We use "_- to show that the strategy is not restricted by the condition (Location, Ball and etc.) in Table 1 .

We define this player as an upper-agent. It is described in Fig. 5.

Assume that this player keeps a ball and his team is in offense. Besides, this player is out of goal area (where (not_goal_area)). And when the distance between him and the opponent goal becomes short, he behaves actually. In this case, this player decides his status (role (pass)). Then, he decides his behavior (shoot) based on decided status (role(pass)) and status of environment (Dist: short). You should notice that this player can shoot the ball. Our upperagent behaves through two steps, deciding a status and deciding an action. For 


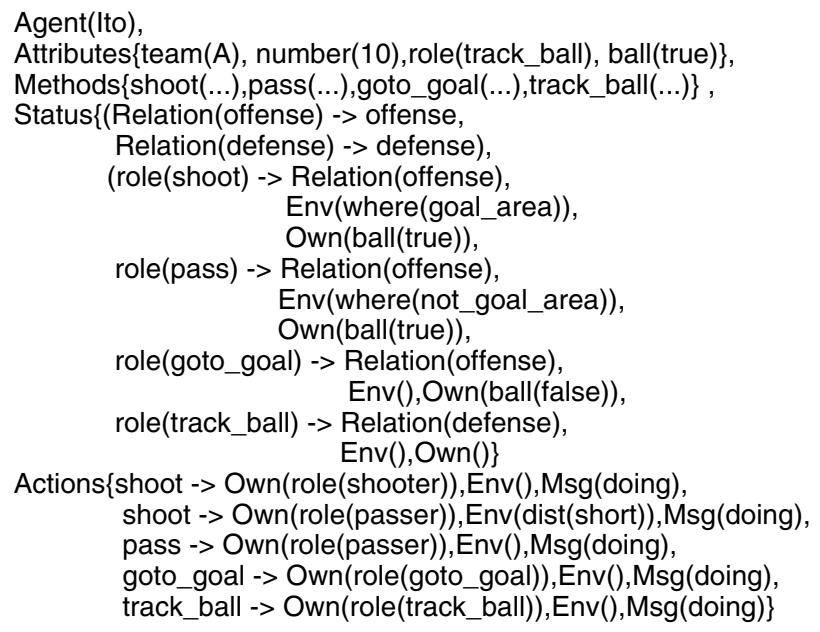

Fig. 5. A Description of A Soccer Player

the second step, he can change his behavior from passing the ball to shooting the ball. It implies that our agent model can represent exceptional behaviors.

\section{A Description-Processing System}

In this section, we describe an implementation of multiple objects. A multiple object consists of some types of agents. Hence, the key is to implement agents. In the current version of the system, it is implemented as an interpreter which may result in a modification of the original specification of the agent.

\subsection{A Common Architecture of Agents}

As we stated in Definition 8, 9, 10 , each agent has some descriptions and one or two processors. Each agent has an interpreter which interprets the descriptions of the agent. Figure. 6 illustrates the common architecture of agents in our system. Each agent basically consists of three units as follows:

1. Communication Unit

This unit is an interface that communicates with other agents, and translates messages coming from other agents into a form that the agent can understand.

2. Specification Unit

This unit is a file that includes attributes, methods, and rules which the agent required in behaving autonomously and pro-actively. 


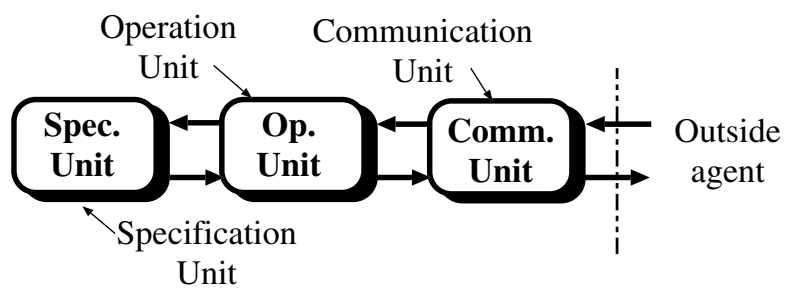

Fig. 6. Common Architecture of Agents

\section{Operation Unit}

This unit is an interpreter that interprets(executes) contents of the specification unit. It also modifies contents of the specification unit and invokes a proper method, possibly.

The operation unit is a kind of interpreter and interprets contents of the specification unit. An agent communicate with other agents via the communication unit in behaving.

\subsection{Class Hierarchy}

Each agent is an instance of a class. Our system contains the following classes:

1. Agent Class

This class owns common properties of agents, which are an agent identity, attributes, and methods.

2. Lower-Agent Class

This class inherits the properties from Agent Class and has an additional property which is an action processor(interpreter).

3. Upper-Agent Class

This class inherits the properties from Lower-Agent Class, and has an additional property which is a status processor(interpreter).

4. Environmental-Agent Class

This class inherits the properties from Upper-Agent Class, and has properties which are method to broadcast and multi-cast in the environmental-agent.

These classes forms an inheritance hierarchy rooted at Agent Class.

For implementing soccer agents easily, a set of subclass are designed.

\section{SoccerEAgent Class}

This class inherits properties from Environmental-Agent Class. As we use this class as interface of the soccer server, this class require method of Input/Output to the soccer server. 


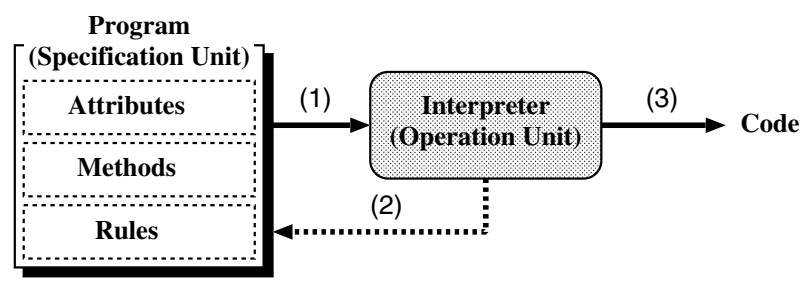

Fig. 7. A Behavior of the operation unit(interpreter)

\section{SoccerUAgent Class}

This class inherits properties from Upper-Agent Class. This class has communication method to SoccerEAgent Class.

3. SoccerLAgent Class

This class inherits properties from Lower-Agent Class. This class also has communication method to SoccerEAgent Class.

In the current implementation, we do not allow communications between upper-agents and lower-agents directly. They communicate through the environmentalagent.

\subsection{The Interpreter of agents}

The interpreter(operation unit) of agents is implemented in Java. It plays the role of a status processor and an action processor. Each agent has an only interpreter, even if the agent like an upper-agent, and an environmental-agent has both of these processors. In this case, the unit behaves according to the description of the agent in two steps. Therefore, this interpreter behaves as illustrated in Fig. 7. The unit of an upper-agent and an environmental-agent behaves in order of $(1) \rightarrow$ $(2) \rightarrow(1) \rightarrow(3)$ in Fig. 7. In (2), the (operation) unit modifies the specification unit(program) according to the rules. Then the operation unit interprets the program again. On the other hand, The unit of an lower-agent behaves in order of $(1) \rightarrow(3)$.

\section{Experiments}

To confirm the efficiency of our model and system, two sets of experiments are designed. One is to compare the power of different teams which are consisted of different type players. The other is to let our team attend the RoboCup Japan Open held in Tokyo, Japan, recently.

\subsection{Designs and Experiments in Our System}

First, three primary types of soccer players are designed. 
Table 2. Hybrid-Player Team(HPT) vs. Upper-Player Team(UPT)

\begin{tabular}{|c|c|c|c|c|c|c|c|c|c|c|c|}
\hline \multicolumn{6}{|c|}{ Hybrid-Player Team kicks off. } & \multicolumn{6}{|c|}{ Upper-Player Team kicks off. } \\
\hline HPT & JPT & result & HP? & JPT & \begin{tabular}{|l} 
result \\
\end{tabular} & $\mathrm{HP}^{\prime}$ & UPT & \begin{tabular}{|l|l|} 
result \\
\end{tabular} & $\mathrm{HP}^{r}$ & $\overline{\mathrm{UPT}}$ & result \\
\hline 3 & 1 & $\begin{array}{l}\mathrm{HPT} \\
\end{array}$ & 4 & 3 & HPT & 4 & 1 & HPT & 4 & 0 & HPT \\
\hline 3 & 1 & HPT & 3 & 1 & HPT & 4 & 1 & HPT & 7 & 1 & HPT \\
\hline 2 & 0 & HPT & 6 & 3 & HPT & 4 & 1 & HPT & 3 & 4 & UPT \\
\hline 4 & 1 & HPT & 5 & 4 & HPT & 2 & 2 & DRAY & 5 & 0 & HPT \\
\hline 3 & 4 & UPT & 4 & 5 & UPT & 8 & 1 & HPT & 4 & 2 & HPT \\
\hline 3 & 1 & HPT & 4 & 4 & DRAY & 4 & 1 & HPT & 3 & 2 & HPT \\
\hline 3 & 4 & UPT & 3 & 1 & HPT & 5 & 1 & HPT & 4 & 2 & HPT \\
\hline 4 & 3 & HPT & 5 & 6 & UPT & 3 & 3 & DRAY & 2 & 2 & DRAW \\
\hline 2 & 2 & DRAW & 8 & 1 & HPT & 6 & 4 & HPT & 2 & 1 & HPT \\
\hline 5 & 1 & HPT & 4 & 6 & UPT & 4 & 2 & HPT & 6 & 2 & HPT \\
\hline 5 & 1 & HPT & 3 & 2 & HPT & 5 & 3 & HPT & 4 & 3 & HPT \\
\hline 2 & 3 & UPT & 4 & 1 & HPT & 2 & 3 & UPT & 3 & 3 & DRAW \\
\hline 6 & 1 & HPT & & & & 5 & 2 & HPT & & & \\
\hline
\end{tabular}

Winning ratio of HPT : 0.72

Winning ratio of UPT : 0.16

\section{Lower-Player}

This player consists of lower-agent and environmental-agent, and behaves reflectively using lower-agent.

2. Upper-Player

This player consists of upper-agent and environmental-agent, and behaves based on status decided by status processor.

3. Hybrid-Player

This player consists of upper-agent, lower-agent, and environmental-agent.

This agent autonomously behaves based on status decided by status processor or behaves reflectively.

We then generate three teams using these players: Lower-Player Team, UpperPlayer Team, and Hybrid-Player Team. We simulates games using these three teams.

Table 2 shows the results of matches Hybrid-Player Team (HPT) and UpperPlayer Team (UPT). HPT wins the games at winning ratio 0.72. In HPT, when the upper-agent does not behaves in $100 \mathrm{~ms}$ intervals, the lower-agent behaves reflectively. The number of behaviors of the upper-agent is 5 times of that of the lower-agent in the games. On the other hand, only the upper-agent behaves autonomously in UPT. The cause of win of HPT is that HPT behaves faster than UPT exactly.

Table 3 shows the results that Hybrid-Player Team (HPT) competes LowerPlayer Team (LPT). HPT does not lose the games. All Lower-Players traced the 
Table 3. Hybrid-Player Team(HPT) vs. Lower-Player Team(LTP)

\begin{tabular}{|c|c|c|c|c|c|c|c|c|c|c|c|}
\hline \multicolumn{6}{|c|}{ Hybrid-Player Team kicks off. } & \multicolumn{6}{|c|}{ Lower-Player Team kicks off. } \\
\hline \multicolumn{6}{|c|}{ HPT LPT/result|HPT LPT resplt } & \multicolumn{2}{|c|}{ HPT LPT } & \multirow{2}{*}{$\begin{array}{c}\text { result } \\
\text { HPT }\end{array}$} & \multicolumn{2}{|c|}{ HPT LPT } & result \\
\hline 6 & 3 & HPT & 5 & 1 & $\overline{\mathrm{HPT}}$ & 5 & 2 & & 6 & 1 & HPT \\
\hline 6 & 3 & HPT & 5 & 2 & HPT & 5 & 3 & HPT & 4 & 2 & HPT \\
\hline 7 & 3 & HPT & 5 & 2 & HPT & 5 & 1 & HPT & 5 & 2 & HPT \\
\hline 6 & 3 & HPT & 5 & 3 & HPT & 4 & 4 & DRAW & 4 & 2 & HPT \\
\hline 7 & 1 & HPT & 5 & 2 & HPT & 5 & 1 & HPT & 5 & 3 & HPT \\
\hline 5 & 3 & HPT & 7 & 2 & HPT & 6 & 2 & HPT & 4 & 3 & HPT \\
\hline 5 & 2 & HPT & 4 & 4 & DRAW & 5 & 3 & HPT & 4 & 1 & $\mathrm{HPT}$ \\
\hline 6 & 3 & HPT & 5 & 1 & HPT & 5 & 3 & HPT & 5 & 2 & HPT \\
\hline 6 & 3 & HPT & 7 & 2 & HPT & 4 & 1 & $\mathrm{HPT}$ & 5 & 3 & $\mathrm{HPT}$ \\
\hline 5 & 2 & HPT & 6 & 2 & HPT & 6 & 3 & НPT & 4 & 2 & HPT \\
\hline 7 & 2 & HPT & 5 & 2 & HPT & 4 & 4 & DRAW & 7 & 3 & HPT \\
\hline 4 & 2 & HPT & 5 & 2 & HPT & 6 & 2 & $\mathrm{HPT}$ & 5 & 1 & HPT \\
\hline 6 & 3 & HPT & & & & 4 & 2 & HPT & & & \\
\hline
\end{tabular}

Winning ratio of HPT : 0.94

Winning ratio of LPT $: 0.00$

ball, and gathered around the ball. On the other hand, Players of HPT spread in the soccer field and behave autonomously.

We do not show that Upper-Player Team competes Lower-Player Team. The result of the games is similar to the result of the matches HPT and LPT. UPT wins the games in a high rate.

From the above results, we conclude that HPT is the strongest team in our experiments. It implies that the ability of agents who can behave appropriately in a fixed interval is very important in RoboCup.

Our system has another more important advantage. From a real point of view, it is necessary to modify the strategies of the upper/lower-agents dynamically in the game. In our system, the environmental-agent are behaved as the interface between the soccer server and upper/lower-agents. In the other words, the environmental-agents can be viewed as soccer players, and the upper/loweragents are the inference engine of the environmental-agents. Therefore, it is possible to modify upper/lower-agents dynamically in the game. It implies that our system is very useful when modifying soccer players was repeated to improve team plays and strategies.

\section{$5.2 \quad$ NIT Stones 98}

For comparing with the other teams that are implemented in different model and architecture, we took part in in the RoboCup Japan Open which held on April 9-11, 1998 in Tokyo, Japan. We implemented our team, NIT Stones, as 
a hybrid-player team. We qualified in the trial heats and finally entered into semifinals. As the result, we are the third winner among all 10 teams. The detail of the result are shown in the following URL(in Japanese):

Trial http://www.er.ams.eng.osaka-u.ac.jp/robocup/jnc/events/jopen98/jopenresult-sim.html

Final http://www.er.ams.eng.osaka-u.ac.jp/robocup/jnc/events/jopen98/jopenresult-sim2.html

Our team played faster and more exact than other teams in the competitions. It implies that our model and system are effective in dynamic environment like soccer games.

\subsection{Results of RoboCup-98}

JDK-1.2 $\beta 3$ was installed in the Workstations of RoboCup-98. However, we implemented our team with JDK-1.1.x. To our great regret, our players froze in the games frequently, although they had never frozen before RoboCup-98. As stated above, our team had a good performance and was awarded third prize in Japan Open 98 . The $\beta$ version system may have been the cause that our players froze.

In these games, we have observed that our players behaved faster and more accurate than other teams. It shows that our model is efficient for the given challenge. In the next RoboCup, we must confirm that our players can behave well in various systems. Besides, we look forward to build up better team plays.

\section{Conclusion}

In this paper, we applied the agent-oriented paradigm to represent multiple objects and a new agent model, EAMMO, is proposed. Besides, we also proposed a description-processing system for EAMMO. We confirmed that our agent model and system are applicable to soccer agents as multiple objects in RoboCup through our simulations. We also confirmed that EAMMO can represent realtime multi-agents systems in our simulations (Hybrid-Player Team). Our system has another more important advantage. From a real point of view, it is necessary to modify the strategies of the upper/lower-agents dynamically in the game. In our system, the environmental-agent are behaved as the interface between the soccer server and upper/lower-agents. In other words, the environmental-agents can be viewed as soccer players, and the upper/lower-agents are the inference engine of the environmental-agents. Therefore, it is possible to modify upper/loweragents dynamically in the game. It implies that our system is very useful when soccer players are modified frequently to improve team plays and strategies. This effect is also confirmed by the result of our team in the competitions of the RoboCup Japan Open.

Our current system is an interpreter-based system implemented in Java. It makes our system behavior a little bit slowly. To improve the performance of 
our system, we plan to introduce a translator which generates Java code from the specification units. Moreover, we will use threads more in implementing our future system.

\section{References}

1. J. Runbaugh, M. Blaha, W. Premerlani, F. eddy, W. Lorensen: Object-Oriented Modeling and Design, Prentice Hall(1991).

2. J. J. Shilling: "Three Steps to Views: Extending the Object-Oriented Paradigm", OOPSLA '89 Proceedings, (1989) 353-361.

3. J. Richardson, P. Schwarz: "Aspects: Extending Objects to Support Multiple, Independent Roles", ACM SIGMOD '91, (1991) 298-307.

4. A. Albano, R. Bergamini, G. Ghelli, R. Orsini: "An Object Data Model with Roles" Proceedings of the 19th VLDB Conference, (1993) 39-51.

5. Y. Kambayashi, Z. Peng: "Object Deputy Model and Its Applications" Proceedings of Fourth International Conference on Database System for Advanced Applications DASFAA '95, (1995) 1-15. 1995.

6. Y. Shoham: "Agent Oriented Programming" Artificial Intelligence, 60(1), (1993) $51-92$.

7. F. G. $\mathrm{M}^{c}$ Cabe, K. L. Clark: "April - Agent PRocess Interaction Language" Intelligent Agents, Lecture Notes in Artificial Intelligence 890, (1995) 324-340.

8. M. J. Wooldridge, N. R. Jennings (Eds.): Intelligent Agents, Lecture Notes in Artificial Intelligence 890, (1995).

9. S. Janson, S. Haridi: Programming Paradigms of the Andrra Kernel Language, SICS Research Report R91:08, (1991). 